\title{
The first thoracic triumvirate
}

\author{
Nicholas Hart, ${ }^{1}$ Gisli Jenkins, ${ }^{2}$ Alan Smyth ${ }^{3}$
}

An editorial board delivering basic science, translational science and clinical trials across paediatrics and adults

We are incredibly proud and honoured to accept the position of Editors-in-Chief of Thorax. Thorax is the premier respiratory journal in the UK, and the official journal of the British Thoracic Society (BTS). It currently has an impact factor of 8.29, making it the third highest ranked respiratory journal in the world. We believe that Thorax has for many years reflected the strength of the British respiratory community publishing wideranging, high-calibre research across the translational spectrum. This view is supported by metrics showing that the UK has the highest $\mathrm{H}$ factor within Europe and the second highest $\mathrm{H}$ factor in the world after the USA. Furthermore, the UK is the third most cited country in the world, after the USA and China. The strong association with the BTS has been a major strength of the Journal because it represents the clinicians, clinical academics and scientists within respiratory medicine in the UK. We have 'grown up' with Thorax and the BTS and have seen how the Journal has grown from strength to strength under the strong, astute editorial guidance of a number of outstanding clinical academics, most recently Professors Andy Bush and Ian Pavord, and we are grateful to them for all the hard work they have done to ensure the continued success of the Journal.

In the rapidly evolving world of research dissemination and globalisation, we are acutely aware of the challenges that lie ahead to ensure the continued success of Thorax and the academic respiratory community. In the age of communication and information technology the world is a much smaller place and collaboration is as likely to occur across borders as institutional departments.

\footnotetext{
'Lane Fox Respiratory Service, St Thomas' Hospital, Guy's \& St Thomas' NHS Foundation Trust, UK; ${ }^{2}$ Centre for Respiratory Research, University of Nottingham, Nottingham, UK; ${ }^{3}$ Division of Child Health, Obstetrics \& Gynaecology, University of Nottingham, Nottingham, UK

Correspondence to Dr Nicholas Hart, Lane Fox Respiratory Service, St Thomas' Hospital, Guy's \& St Thomas' NHS Foundation Trust, London SE1 7EH, UK; nicholas.hart@gstt.nhs.uk
}

Likewise the dissemination and impact of data generated needs to be relevant throughout the world. The proliferation of new journals, most notably The Lancet Respiratory Medicine, and alternative metrics means that this is an exciting time to take over at the helm of Thorax and we are looking forward to the challenge immensely.

Our editorial policy aims to build upon the strengths that previous Editors have identified and introduce some changes that will ensure the success of the Journal and meet the needs of the academic community. Our ultimate goal is to prioritise the highest quality translational research and enhance the scope of Thorax by publishing more cutting edge basic science through to clinical trials and the highest quality systematic reviews. To encourage publication of high quality research papers we have increased the word limit for 'Original Research' to permit extra data and methodology to be included to promote the fundamental principles of repeatability and reproducibility. Similarly we have shifted the focus of the 'Letters Section' to encourage submission of letters containing data that replicates, or refutes, previously published data. We have therefore increased the word and data limits for submitted research letters. We will continue to publish clinically relevant manuscripts such as 'Case Based Discussions' and 'Images in Clinical Medicine' and high quality educational content but we will not pursue the publication of clinical audit and opinion letters.

A key strategic aim of the Journal is enhanced internationalisation of submissions, content and relevance of published manuscripts and educational content, and our Deputy Editors Professors Naftali Kaminski and Margaret Rosenfeld, from the USA, will be central to this process. To showcase recent advances in translational research from around the globe we will be commissioning a 'Best of' series starting with 'Best of the USA' in 2016 and a novel 'Guidelines in Context' series that will ensure national guidelines are framed in an internationally relevant context. To promote novel ways of delivering on-line content we ask authors to provide a 140 character 'Twitter' summary of their manuscript and have recruited Editorial Board members with specific social media expertise.

To enhance the service we provide to authors, we will streamline the review process, and offer protocol review of clinical trials and we will also be piloting protocol review of basic science studies. The goal is to get high quality research published as quickly as possible and return manuscripts to authors that would be best placed in another journal for resubmission as quickly as possible. We will also offer the opportunity of automatic resubmission of manuscripts declined on the basis of priority to other $B M J$ group journals, primarily BMJ Open Respiratory Research. We will guide Thorax reviewers to limit major criticisms to three or recommend rejection of the manuscript to reduce the burden of the review process on the authors, the reviewers and the editorial team. We will embed statistical oversight of all manuscripts at the initial stages of the review process to ensure that all data have been appropriately analysed and manuscripts are not delayed by subsequent re-reviews. We are also considering, with the senior team at $B M J$ publishing, the implementation of double blind or open peer review.

We are aware of the time commitment that this places on members of the Editorial Board and reviewers and we are extremely grateful for their contribution to the peer review process without which no publication of any manuscript would be possible. We believe we have assembled an outstanding team of Associate and Statistical Editors ensuring that all key specialised areas of Respiratory Medicine have sufficient expert oversight to facilitate fair and robust peer review. Similarly the Editorial Board members who perform reviews are extremely accomplished academics and we are privileged to work with them on the Journal.

This is a period of considerable change and we hope the formation of the First Thoracic Triumvirate will be able to deliver our aims with more subtlety, and considerably less bloodshed, than triumvirates of days gone by.

\section{Competing interests None declared.}

Provenance and peer review Commissioned; internally peer reviewed.

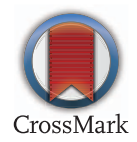

To cite Hart N, Jenkins G, Smyth A. Thorax 2015;70:917.

Thorax 2015;70:917.

doi:10.1136/thoraxjnl-2015-207756 